\title{
Effects of home reading training on reading and quality of life in AMD—a randomized and controlled study
}

\author{
K. Kaltenegger ${ }^{1} \cdot$ Stephan Kuester $^{1}$ (1) - E. Altpeter-Ott ${ }^{2}$ - G. W. Eschweiler ${ }^{3}$ - A. Cordey ${ }^{1,2}$ • I. V. Ivanov ${ }^{1} \cdot$ P. Martus ${ }^{4}$. \\ C. Knipp ${ }^{1} \cdot$ S. Trauzettel-Klosinski ${ }^{1}$
}

Received: 7 February 2019 /Revised: 9 April 2019 / Accepted: 16 April 2019 / Published online: 20 May 2019

(C) The Author(s) 2019

\begin{abstract}
Background Age-related macular degeneration (AMD) causes reading impairment, reduced quality of life (QoL), and secondary depression. We have shown that support with magnifying aids improved reading speed (RS), emotional and cognitive status, and QoL. The present study investigates whether additional reading training (RT) (after adapting to appropriate visual aids) can further improve vision rehabilitation.

Methods Patients with dry AMD were randomly assigned to 2 groups. The primary RT group (P-RTG, $n=25$ ) trained with sequentially presented text (RSVP), and the control group $(\mathrm{CG}, n=12)$ performed placebo training (crossword puzzles) and later crossed over to RT, so that altogether 37 participants performed reading training. Patients trained at home on a PC for 6 weeks. RS was assessed during reading printed paragraphs of text aloud. Using a scanning laser ophthalmoscope, we examined fixation stability and preferred retinal locus (PRL) for fixating a cross, as well as PRL and eye movements during reading single words. We assessed emotional status by Montgomery-Åsberg Depression Rating Scale (MADRS), cognitive status by dementia detection test (DemTect) and QoL by Impact of Vision Impairment (IVI) profile. Visual acuity and magnification requirement were examined by standard procedures. All variables were measured before and after placebo training, before and after RT, and after 6 weeks without training (follow-up).

Results RS improved significantly in the P-RTG during RT, but not in the CG during placebo training. The effect remained stable at follow-up. Fixation performance and eye movement variables did not change. Emotional status (MADRS) improved in P-RTG during RT and showed a significant difference of the change of scores between the 2 groups.

Complete IVI scores improved significantly during RT and remained stable.

Conclusion The results indicate that patients with AMD, who already use magnifying aids, benefit from additional RT and that it can contribute in preventing depression and improve QoL.

Trial registration The study was registered at the German Clinical Trials Register (DRKS00015609).
\end{abstract}

Keywords Age-related macular degeneration $($ AMD) $\cdot$ Vision rehabilitation $\cdot$ Computer-based reading training $\cdot$ RSVP $\cdot$ Reading speed · Quality of life

K. Kaltenegger and S. Kuester share first-authorship.

Stephan Kuester

Stephan.kuester@uni-tuebingen.de

1 Vision Rehabilitation Research Unit, Center for Ophthalmology, University of Tübingen, Tübingen, Germany

2 Low Vision Clinic, Center for Ophthalmology, University of Tübingen, Tübingen, Germany

3 Section Geriatric Psychiatry, Department for Psychiatry and Psychotherapy, University of Tübingen, Tübingen, Germany

4 Institute for Clinical Epidemiology and Applied Biometry, University of Tübingen, Tübingen, Germany

\section{Introduction}

Reading is a key function for participation in the society. It improves the quality of life (QoL) by enhancing independence, communication, and cognitive agility. Loss of the ability to read is often caused by central vision loss due to agerelated macular degeneration (AMD). It is the most common cause of severe visual impairment in the Western world [1] and can reduce the QoL $[2,3]$.

Several studies have shown that AMD patients have a higher rate of depression compared with the general population [3-6], with a prevalence of 2-5\% [7]. The development of depression can also lead to subjectively reduced visual 
function in these patients [6] and to reduction of cognitive abilities [8]. This is why vision rehabilitation of AMD patients is very important, as we have shown that its effectiveness is $94 \%[9,10]$. In a previous pilot study, we also showed that support with magnifying aids improved reading speed (RS), emotional and cognitive status, and QoL [11].

The current randomized, controlled study investigates whether additional reading training (RT) for AMD patients, after they have adapted to appropriate visual aids, can further improve the results of vision rehabilitation. This seemed likely, because we had found in a previous study on patients with juvenile maculopathy (M. Stargardt) that two kinds of RT could cause a significant increase of RS: rapid serial visual representation (RSVP) and an oculomotor training [12].

Thus, the purpose of the current study was to provide answers to the following questions:

Can additional RT after adaptation to optimal visual aids improve RS in patients with AMD?

Can RT using RSVP lead to improved fixation stability?

Can RT improve emotional well-being?

Can RT have a positive effect on vision-related QoL?

This is the first randomized and controlled trial (RCT) on computer-based RT at home for patients with AMD. Previous RT studies in AMD patients did either not use a control group [13] or did not include training at home [14]. One step in the same direction was the work by Coco-Martin et al. (2013) [15], where home training was effective, but not computerbased, and required several visits at the clinic for customizing the degree of task difficulty.

First results of this study were presented at ARVO 2016 and at the Meeting of the German Ophthalmology Society (DOG) $2016[16,17]$.

\section{Methods}

\section{Study design}

We screened 481 AMD patients, and 52 who met the inclusion criteria were considered for the study. Fifteen patients dropped out after inclusion. The reasons were as follows: decreasing visual acuity (2), increasing magnification requirement (3), change to wet AMD which required anti-VEGF treatment (1), RS value not documented after RT (1), personal reasons (6), suspected dementia in a dementia detection test (DemTect) (1), and unknown (1). Ultimately, 37 patients participated (see Fig. 1), who were randomly assigned to one of two groups: a primary RT group (P-RTG, $n=25$ ), who started directly with RT, and a control group (CG, $n=12$ ).

The CG received a placebo training. At first, they served as a "waiting group" and received the RT later. This step was necessary, because the recruitment of the patients was very difficult: the majority of the screened AMD patients either had too advanced a stage of the disease (magnification requirement $>10$-fold), or their AMD had changed to the wet condition, or they participated in other treatment studies at the same time.

The main outcome variable was reading speed (RS).

There were no statistically significant differences between the groups at baseline regarding age, RS, visual acuity, magnification requirement, and disease duration.

The 12 patients of the $C G$ received 6 weeks of placebo training (from $\mathrm{t} 0-\mathrm{t} 1$ ), before they started the RT (from t1-t2). All examinations were performed at all visits. Therefore, altogether 37 patients participated in the RT. This group will be referred to below as the "total RT group" (T-RTG). The T-RTG was examined before training (at $\mathrm{t} 1$ ), directly after the 6 weeks of training (t2), and 6 weeks after the end of training (t3).
Fig. 1 Study design. The patients were randomly assigned to either the control group (CG), who solved crossword puzzles as placebo training, or to the primary RT group (P-RTG), who performed the RSVP training. The 12 patients of the $C G$ served as a waiting group to perform the $\mathrm{RT}$ after the placebo training. In total, 37 patients received the RT (total RT group T-RTG)

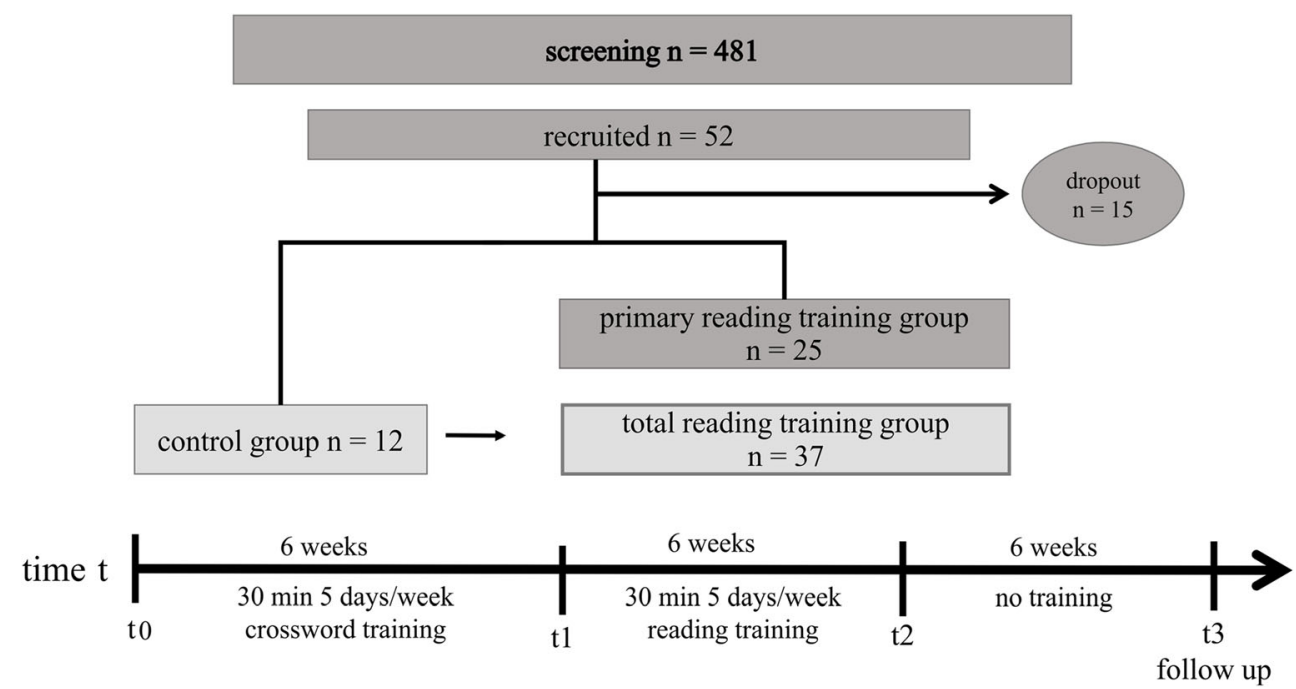




\section{Participants}

We recruited 37 AMD patients $(57 \%$ women, median age 72 years, IQR 67.5-79.0) from the Center of Ophthalmology, University of Tübingen, Germany, and from private ophthalmologists' offices in the region.

\section{Baseline data}

Median visual acuity was $0.70 \operatorname{logMAR}$ (IQR $0.52-0.80$ ), 0.20 decimal (IQR 0.16-0.30). Median magnification requirement was 3.0-fold (IQR 2.3-6.0). Median disease duration $(n=33)$ was 4 years (IQR 1.5-8.0). For 4 patients, disease duration could not be determined.

Inclusion criteria Dry AMD with an absolute central scotoma including the foveola, (small parafoveal seeing islands were permitted), non-foveal (parafoveal or eccentric) fixation, magnification requirement $1.25-10$-fold, visual acuity $0.2-1.1$ $\log$ MAR (decimal 0.08-0.60), previous adaptation to the use of magnifying aids of at least 6 weeks.

Exclusion criteria Any other eye disease, exudative AMD, simultaneous anti-VEGF or other treatments, markedly reduced emotional and cognitive status that could have jeopardized participation in the study.

\section{Examinations}

An overview of all examinations is shown in Table 1.

\section{Ophthalmological examination}

This included distance visual acuity (ETDRS charts), magnification requirement (Zeiss reading charts), eye morphology, and visual fields (Tübingen Manual or Automated Perimetry).

The better eye had to fulfill the inclusion criteria.

If a patient did not use an optimal magnifying aid (optical or electronic), the appropriate aid was prescribed, which the patient had to use for at least 6 weeks before inclusion in the study.

\section{Reading speed}

RS of printed text (page reading) was the main outcome variable and was measured in words per minute (wpm) using the German version of the International RS Texts (IReST) $[18,19]$ at $\mathrm{t} 0, \mathrm{t} 1, \mathrm{t} 2$, and $\mathrm{t} 3$. For this purpose, the patients read one IReST paragraph aloud either binocularly or monocularly using the better eye, with their individual magnifying aid. For the repeated measurements of RS, we used different paragraphs of the same performance category, so that each one was used only once. It is important to note that the patients performed the RT at home on a laptop computer with single word presentation (RSVP, details see below), while the training effect on RS was measured in the clinic by reading IReST paragraphs as printed text like in an everyday situation. This allowed testing whether training effects generalize to everyday reading and remain stable [20].

In addition, RS during the RSVP training (RSVP-RS) was documented by the training software at the beginning and end of the training.
Table 1 Examinations and schedule during the study. $C G$ control group, FSI fixation stability index, $P R L$ preferred retinal locus, $M A D R S$ Montgomery-Åsberg Depression Rating Scale, DemTect $\mathbb{B}$ dementia detection test, $Q o L$ quality of life

\begin{tabular}{|c|c|c|c|c|c|c|c|}
\hline Examinations & $\begin{array}{l}\text { t0 } \\
\mathrm{CG}\end{array}$ & $\begin{array}{l}\text { Placebo } \\
\text { training CG }\end{array}$ & $\mathrm{t} 1$ & $\begin{array}{l}\text { RSVP training at } \\
\text { home }\end{array}$ & $\mathrm{t} 2$ & $\begin{array}{l}\text { Pause without } \\
\text { training }\end{array}$ & $\mathrm{t} 3$ \\
\hline Clinical examination & + & & + & & & & \\
\hline $\begin{array}{l}\text { Reading speed of printed } \\
\text { text (IReST) }\end{array}$ & + & & + & & + & & + \\
\hline RSVP-RS & & & + & & + & & \\
\hline Eye movements (SLO): & & & + & & + & & + \\
\hline \multicolumn{8}{|l|}{ FSI and PRL for a cross } \\
\hline \multicolumn{8}{|l|}{$\begin{array}{l}\text { PRL and eye movement } \\
\text { variables }\end{array}$} \\
\hline \multicolumn{8}{|l|}{$\begin{array}{l}\text { During reading single } \\
\text { words }\end{array}$} \\
\hline Questionnaires: & + & & + & & + & & + \\
\hline \multicolumn{8}{|l|}{ Depression (MADRS) } \\
\hline \multicolumn{8}{|l|}{ Cognition (DemTect) } \\
\hline \multicolumn{8}{|l|}{ QoL (IVI) } \\
\hline Time & & 6 weeks & & 6 weeks & & 6 weeks & \\
\hline
\end{tabular}




\section{Eye movements}

We collected a supplementary data set that was used to investigate the question whether the training caused a change in:

- fixation stability,

- the retinal locus used for fixation of a cross,

- the retinal locus used for reading a single word, and

- reading time and eye movements during reading a single word (see below).

We used a scanning laser ophthalmoscope (SLO, model 101, Rodenstock, Munich). This method allows simultaneous viewing of the image of the retina and the stimulus to determine the absolute position of the foveola relative to the stimulus [21]. The SLO data were measured in 25 patients. The data from the remaining 12 patients had to be excluded because they were either incomplete or compromised by insufficient image quality.

\section{Fixation stability}

Fixation stability while fixating a cross of $1^{\circ}$ diameter for $20 \mathrm{~s}$ was measured by calculating the fixation stability index (FSI) by the formula
$\mathrm{FSI}=1-\frac{\text { number of different pixel coordinates of a sequence }}{\text { number of all pixel coordinates of a sequence }} \times 100 \%$

The higher the FSI, the more stable the fixation. In a previous study, normal subjects showed an FSI of $91.5 \%$ (81.696.2\%) [22].

Fixation stability is often calculated as the bivariate contour ellipse area (BCEA) $[23,24]$. The FSI can be converted to BCEA by a formula shown in Reinhard et al., 2007 [22].

\section{Retinal locus for fixation of a cross}

As the position of the foveola often cannot be determined morphologically in AMD, the calculation of the position was performed according to Rohrschneider [25]. The preferred retinal locus for fixation (PRL) was assessed as coordinates and then related to the foveolar position (see Fig. 2). Changes of the PRL were assessed by the movement of the fundus, which was indicated by the position of a reference point on a vessel branching using custom image detection software [22]. We measured the distance of the PRL to the foveola in degrees and assigned the PRL location to the quadrants around the fovea (upper, lower, right, and left).

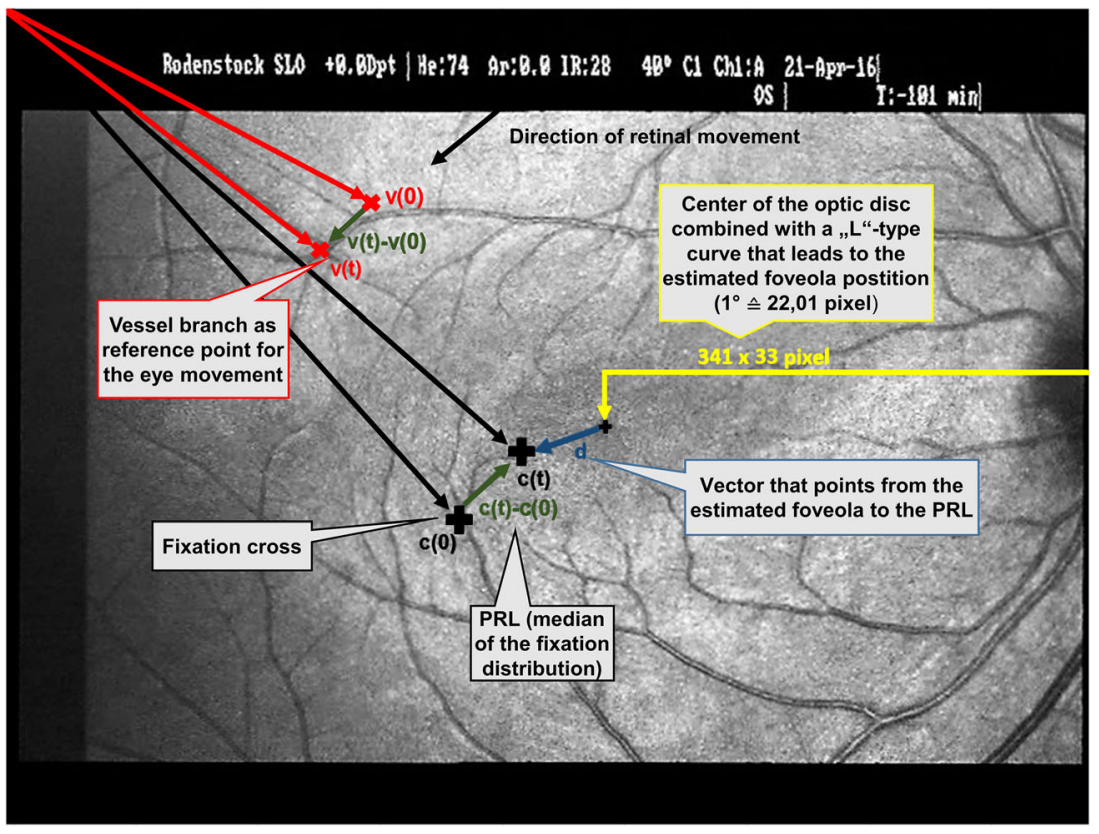

Fig. 2 Schematic diagram of the analysis procedure after Reinhard et al. 2007 [22]. Measurement of the coordinates to determine the preferred retinal locus for fixation (PRL) and the fixation stability index (FSI). The PRL was assessed as coordinates and then related to the foveolar position. The reference region shows the tracking coordinates at a prominent region (e.g., vessel bifurcation) at time $0(\mathrm{v}(0))$ and the time immediately thereafter $(v(t))$. From this, a vector can be calculated $(v(t)-v(0))$, which reflects the direction and size of the retinal movement while fixating the cross. The actual retinal location for fixation of the cross is the point at which the patient views the cross, once at time $0(\mathrm{c}(0))$ and immediately thereafter $(\mathrm{c}(\mathrm{t}))$. A vector can also be calculated between these two points $(\mathrm{c}(\mathrm{t})-\mathrm{c}(0))$, which is equivalent to the vector of the reference region, but exactly in the opposite direction. Below, this vector $(c(t)-c(0))$ is referred to as the "PRL distance (from the fovea)." Since the fixation cross is always at the same coordinate point on the mask of the SLO and the retina moves in the rigid image window, the actual cross coordinates can be determined from the retinal movements 


\section{Reading single words assessed by SLO}

We tested the hypothesis that fundamental characteristics of eye movements during reading single words might show correlations with the observed changes in RS. For this purpose, we recorded the eye movements while reading single words using an SLO in 17 of our patients. We then compared the following variables that were measured before and after the training ( $\mathrm{t} 1-\mathrm{t} 2)$. These variables were as follows: the average time between word presentation and articulation, i.e., the time the patient looked at the word, the number of forward and backward saccades, and the number of fixations.

Single words of 4-5 letters were presented in the letter size of the individual magnification requirement, which was determined during the clinical pre-examination.

While the patients fixated a cross of 36 arcmin diameter, a single word was presented as centered on the fixated position. The patient had to read the word aloud. This was followed by another cross and another word. The same letter size was also used during the training and corresponded to the clinically assessed magnification requirement and the magnifying aid in everyday reading and during the IReST reading test. The PRL during reading single words was compared with the fixation locus for the cross.

\section{Training}

We provided the participants with laptop computers with preinstalled RT software. The RT program displayed the text on the screen as a sequence of single words, one at a time, by rapid serial visual presentation (RSVP) [26-28]. The patients could choose different texts from different categories (nature, culture, sports, and fairy tales), which were stored on the computer. The letter size was preselected according to the individual magnification requirement that was determined during the clinical examination before the beginning of the training. The speed of word presentation was tested at t1 and aimed to achieve a balance between the highest possible speed and best reading comfort for the patient. The CG received placebo training that consisted of solving printed crossword puzzles.

Both groups performed the training exercises at home for 6 weeks, 5 days/week, $30 \mathrm{~min} /$ day (documented by the patients during placebo training and by the software during RT). The 12 patients of the CG transitioned to the RT after finishing the placebo training.

After the 6 weeks of RT (t2), there was a pause of 6 weeks without training, which was followed by another examination (t3) in order to examine whether a potential training effect had remained stable.

\section{Questionnaires}

Score definitions and statistical results are shown in Table 2.

\section{Degree of depression}

To assess the severity of depression, we used the Montgomery-Åsberg Depression Rating Scale (MADRS) [29-31]. The MADRS is especially used in elderly subjects and has several cut-offs. The cut-offs are 0 to 6 , no depression/ symptoms absent; 7 to 19 , mild depression; 20 to 34 , moderate depression; and more than 34 , severe depression. This test is especially suited for detecting changes of emotional status during interventions.

\section{Cognition}

We used the dementia detection test (DemTect) to assess cognitive performance. This test is mainly based on auditory items and was developed for detection of mild cognitive impairment (MCI) [32]. It has a higher sensitivity than the MiniMental-Status test (MMS) for MCI, and the results can be transposed into MMS score values [33, 34]. We used the DemTect primarily as a screening tool to determine whether a participant had to be excluded because of dementia. Additionally, the parallel form of the test was applied to detect possible variations during the training periods $\mathrm{t} 0-\mathrm{t} 1$ (placebo training) and t1-t2 (RT). Thirteen to eighteen points correspond to an age-adapted normal cognitive performance. A DemTect score of 13 corresponds to a MMS score of 28 and a MOCA [35] score of 25.

\section{Quality of life}

To assess QoL, we used the German version of the Impact of Vision Impairment (IVI) questionnaire [36-38]. It has good reliability for measurements within short time intervals [39]. Therefore, it is especially suited to detect changes during an intervention. We used normalized scores to analyze the outcomes and the subscales of functional and emotional QoL [40].

\section{Statistical methods}

We used IBM SPSS Statistics, version 24, for statistical analyses. We presented all descriptive data as medians and their interquartile range (IQR). If the data were normally distributed, we applied parametric methods for significance testing (paired $t$ test, $t$ test for independent samples). In all other cases, we used non-parametric methods (Wilcoxon test, MannWhitney $U$ test, Friedman test). The values are shown in Table 2. Not all patients participated in the follow-up examination $(n=27)$; the calculations were performed for complete 


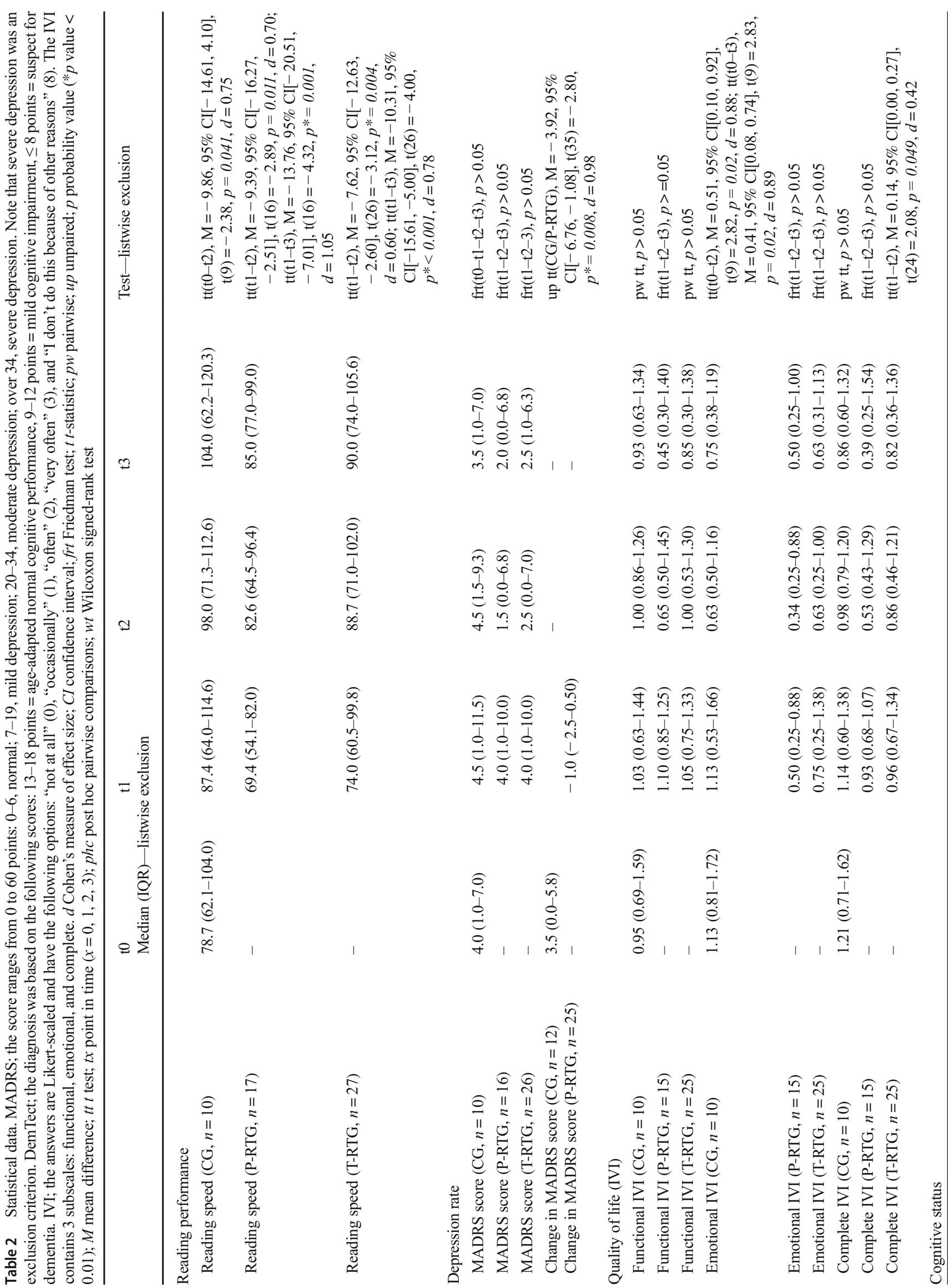


cases (listwise exclusion) without imputation for comparing results between different points in time.

For correlations, we calculated the Pearson correlation coefficient. The threshold level of significance $\alpha$ was 0.05 (twosided) in all statistical tests. A $p$ value of $\leq 0.001$ was defined as "highly" significant.

\section{Results}

\section{Reading speed}

As the data were normally distributed, we applied parametric methods of analysis. The statistical data are summarized in Table 2; the results are displayed in Fig. 3. The group sizes for comparison between the different points in time, including at follow-up (t3), were slightly smaller than for the complete sample, because not all patients participated in the follow-up examination.

In the $\mathrm{CG}$, median RS at t0 was 78.7 wpm, at $\mathrm{t} 187.4 \mathrm{wpm}$, at t2 $98.0 \mathrm{wpm}$, and at $\mathrm{t} 3104.0 \mathrm{wpm}$. The RS increase during placebo training ( $\mathrm{t} 0-\mathrm{t} 1)$ was $8.7 \mathrm{wpm}, 10.6$ during the following RT ( $\mathrm{t} 1-\mathrm{t} 2)$, and 6.0 wpm after the 6 weeks without training (t2-t3). The change from $\mathrm{t} 0$ to $\mathrm{t} 2$ was statistically significant.

In the P-RTG, median RS increased significantly during the RT from t1 (69.4 wpm) to $\mathrm{t} 2(82.6 \mathrm{wpm})$ by $13.2 \mathrm{wpm}$ and remained stable at $\mathrm{t} 3(85.0 \mathrm{wpm})$. The difference from $\mathrm{t} 1$ to $\mathrm{t} 3$ was highly significant.

In the T-RTG, median RS increased significantly from t1 (74.0 wpm) to $\mathrm{t} 2(88.7 \mathrm{wpm})$ by $14.7 \mathrm{wpm}$ and remained stable at $\mathrm{t} 3(90.0 \mathrm{wpm})$. The difference between $\mathrm{t} 1$ and $\mathrm{t} 3$ was highly significant.

Although the general effect on the whole group is only moderate, individual patients benefited considerably: 14 of the 37 patients (38\%) improved their RS by at least $10 \mathrm{wpm}$, which can be considered clinically relevant based on our longstanding clinical experience, and in only $3(8 \%)$ the RS decreased by $\geq 10 \mathrm{wpm}$.

Figure 4 shows the distribution of the change in RS for all patients.

Correlations between change of RS in the T-RTG $(n=37)$ and baseline parameters The improvement of RS correlated negatively with $\mathrm{RS}$ at baseline $(n=37, r=-0.367, p=0.025)$, i.e., patients starting with a lower RS showed more improvement.

No correlations were found between change of RS ( $\mathrm{t} 2-\mathrm{t} 1)$ and the other baseline variables: visual acuity, magnification requirement, age, and disease duration, i.e., no predictive factors were found for improvement of RS.

$\mathrm{RS}$ at $\mathrm{t} 1$ correlated positively with distance visual acuity at t1 $(n=37, r=0.486, p=0.002)$ and with disease duration $(n=$ 
Fig. 3 Median RS for CG and PRTG. CG $(n=10)$ performed placebo (crossword puzzle) training from $\mathrm{t} 0$ to $\mathrm{t} 1$, and $\mathrm{RT}$ from $\mathrm{t} 1$ to $\mathrm{t} 2$. Only the difference between $\mathrm{t} 0$ and $\mathrm{t} 2$ was significant. In the P-RTG $(n=17)$, RS increased significantly during RT (t1 to $\mathrm{t} 2$ ) and remained stable after the 6 weeks without training ( $\mathrm{t} 3$ )

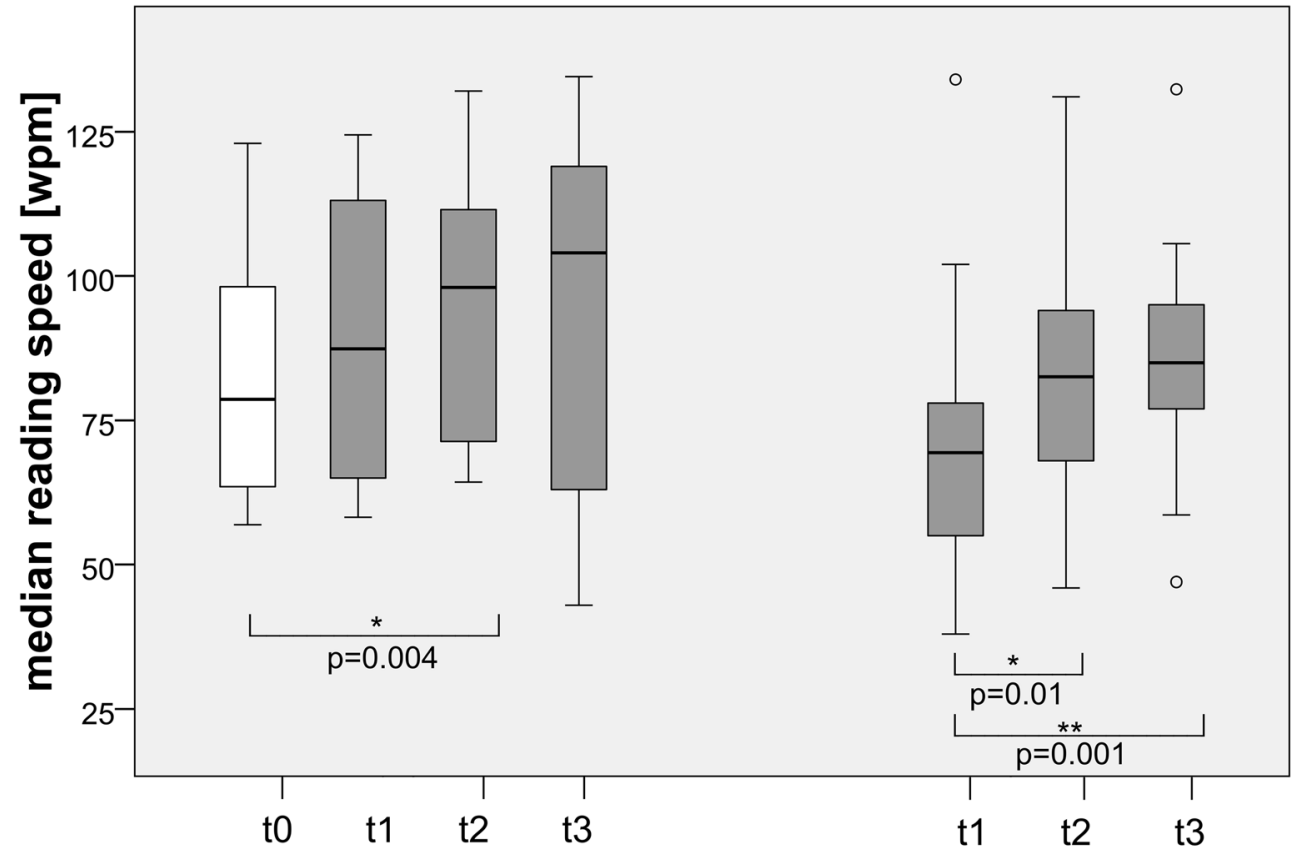

control group primary reading training group
33, $r=0.405, p=0.019)$, but negatively with magnification requirement at $\mathrm{t} 1(n=37, r=-0.419, p=0.01)$.

We looked separately at the subgroup of patients who improved by $\geq 10 \mathrm{wpm}$ and did not find any correlation between the change of RS (t1-t2) with the values at baseline.

RS correlated positively with RSVP-RS (median $=53$ wpm, $r=0.58, n=31, p<0.001$ ) at $\mathrm{t} 1$ and at $\mathrm{t} 2$ (median $=$ 55 wpm, $r=0.4, n=31, p 0.024)$.

\section{Eye movements}

\section{Fixation stability while fixating a cross}

The FSI and the difference of FSI were normally distributed at all points in time and for all groups.

At baseline, the FSI for the CG was $77.8 \%$ (T0; $n=11$ ), for the P-RTG $85.8 \%(\mathrm{~T} 1 ; n=14)$, and for the T-RTG $83.1 \%$ (T1;
Fig. 4 Individual changes in reading speed. The distribution of the individual changes in reading speed during reading training for T-RTG from $t 1$ to $t 2$ is shown

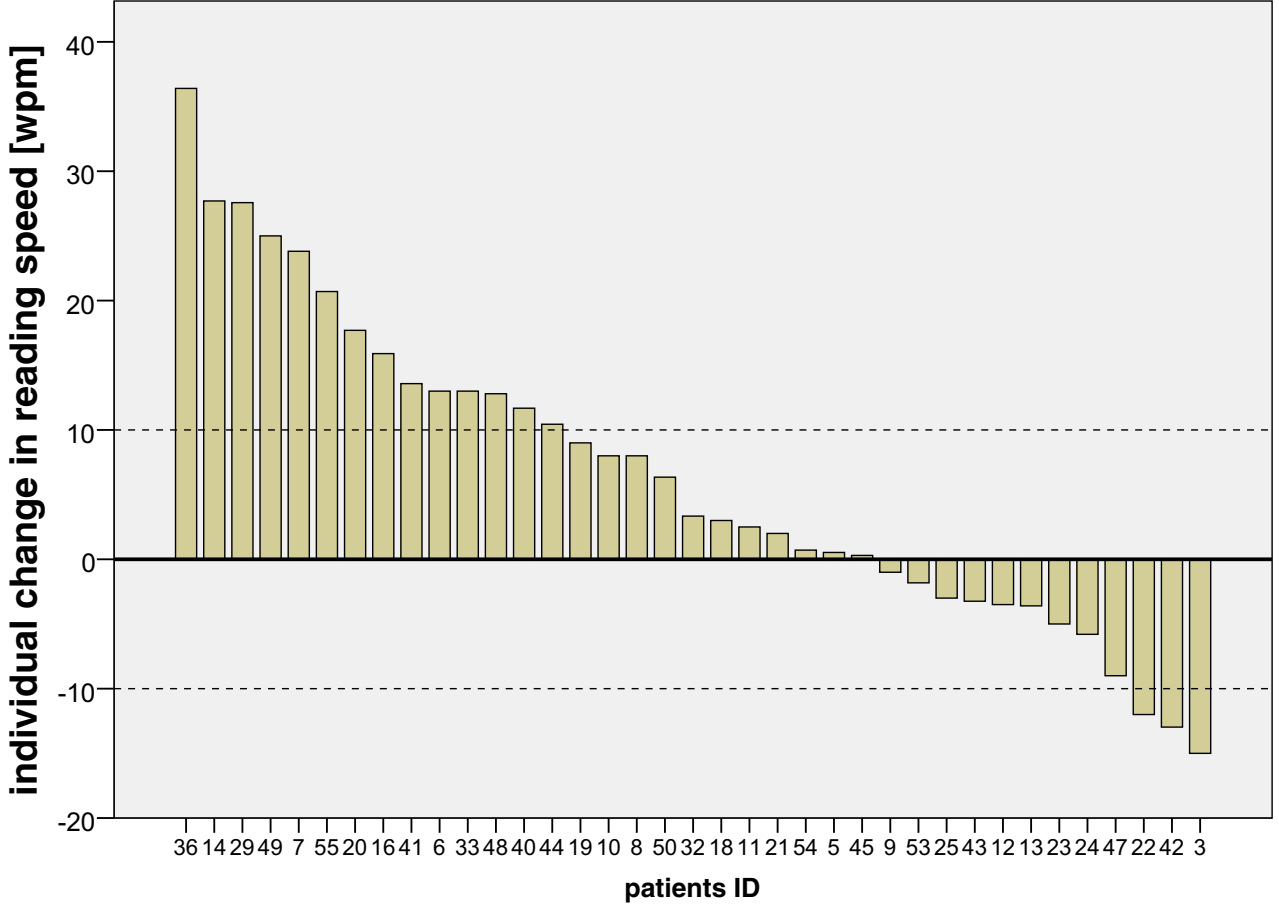


$n=25$ ). The FSI difference between the groups was not statistically significant at baseline.

The FSI did not show any statistically significant changes between the different points in time for all groups. Furthermore, there was no correlation between the change of FSI and the increase of RS pre- vs. post-intervention.

\section{The PRL for fixating a cross}

Most of the patients fixated the cross with a retinal area above the lesion within an area of $6^{\circ}$ radius (at t1, 18 of 25 ; at t2, 22 of 25) and showed only slight changes of the location within this area: the median change of the PRL between $\mathrm{t} 1$ and $\mathrm{t} 2$ (T-RTG, $n=25$ ) was not statistically significant (in the horizontal meridian $0.45^{\circ}$, in the vertical meridian $0.28^{\circ}$ ). A change of location of the PRL by more than $6^{\circ}$ was observed in 4 patients.

\section{Reading single words}

The patients performed an average of 5 fixations per word (median 5.0, IQR 4.25-6.25). None of the tested variables (reading time, number of forward and backward saccades, number of fixations) showed any statistically significant changes brought about by the RT. The result was the same, even if we compared the values between the patients with and without improvement of RS.

We also looked for correlations of the eye movement variables but found them neither with the change in RS during reading of printed text (IReST) nor with the magnification requirement.

\section{Retinal locus for reading single words}

All but two patients used the same PRL for the cross and the words. One patient used an eccentric PRL for words and a paracentral PRL for the cross. Only one patient used different locations for fixating both the cross and the words.

\section{Questionnaires}

The statistical data and definitions are summarized in Table 2.

\section{Emotional status (MADRS)}

Our patients had either only mild or no depressive symptoms at baseline, since severe depressive symptoms were a reason for exclusion. In the CG, the score did not change between different times (see Table 2). In P-RTG, the score improved from 4.0 to 1.5 without reaching significance.
However, the difference between the two independent groups for the change of MADRS score in the CG (t0-t1) vs. the P-RTG (t1-t2) was statistically significant (Fig. 5).

\section{QoL (IVI)}

\section{Complete IVI}

The T-RTG showed a significant improvement during the RT from $\mathrm{t} 1$ to $\mathrm{t} 2$. The $\mathrm{CG}$ did not show any change from to to t1.

Functional IVI The Functional IVI did not show any changes in any group at any time. There were only 2 questions (no. 14 and no. 15) regarding reading (see "Discussion"). The analysis of only these 2 questions did not show any significant differences between the groups.

Emotional IVI In the CG, there was only a slight improvement from to to $\mathrm{t} 1$ and $\mathrm{t} 1$ to $\mathrm{t} 2$, but this became statistically significant for $\mathrm{t} 0-\mathrm{t} 2$ and $\mathrm{t} 0-\mathrm{t} 3$. In the P-RTG, there was no change.

\section{Cognitive status (DemTect)}

The scores were always within the normal range, and there was neither a change in cognitive ability at any time nor a conversion to mild cognitive impairment.

\section{Discussion}

The idea behind the RSVP method is to avoid reading eye movements [26]. Because of the reduced perceptual span in AMD patients [41, 42], they need to make more reading

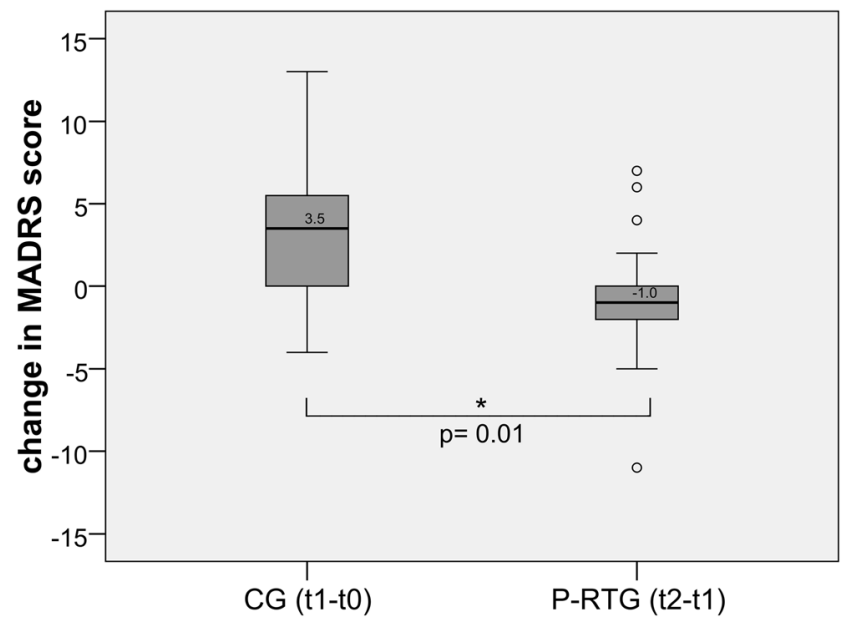

Fig. 5 Change in MADRS score. Change in MADRS score is shown during crossword training from to to $\mathrm{t} 1 \mathrm{CG}, n=10)$ and during reading training from $\mathrm{t} 1$ to $\mathrm{t} 2$ (P-RTG, $n=16$ ). Change to lower values indicates an improvement. The difference in change of MADRS score between the groups is significant (see also Table 2) 
saccades, which is time-consuming [41, 42]. Therefore, eliminating eye movements makes the task easier. The disadvantage is that the patients cannot perform regressions within the text, which is sometimes necessary for text comprehension [43]. Another disadvantage of RSVP is that almost all reading materials in everyday life are in some form of print, but RSVP does not train planning and executing eye movements, which hampers the user if such material needs to be read. This fosters the development of a dependence on computer-aided text presentation. In a former randomized and controlled study, we used two different RT methods to train 36 Stargardt patients: one group received RSVP training, while the other group received oculomotor training, in which the eyes were guided through the line of text by a moving window filled with text. Both groups improved by a mean of $20 \mathrm{wpm}$. Interestingly, the RSVP group improved by shortening the fixation duration and the oculomotor group improved by performing fewer saccades per word [12]. As planning and performing eye movements is time-consuming and more difficult for patients with central vision loss [41], we intended to make the task easier for our AMD patients.

\section{Reading speed}

During RT, median RS improved highly significantly in the PRTG (by $13.2 \mathrm{wpm}$ ) and the T-RTG (by $14.7 \mathrm{wpm}$ ), but not in the $\mathrm{CG}$ (during the placebo training).

Considering a change of $\geq 10 \mathrm{wpm}$ as clinically relevant, we found that 14 patients benefited from the training in a meaningful way but we did not find any predictive factor for future improvement.

The median increase of RS in the T-RTG by $14.7 \mathrm{wpm}$ (t1t2) was less marked compared with that in the Stargardt patients in our previous study, who improved by $20 \mathrm{wpm}$ [12]. This might have been caused, at least in part, by the much higher age of the AMD patients. It should be considered that the main effect in improving RS in AMD is based on magnifying aids $[9,10]$. We showed in a previous RCT that RS, scales of depression, QoL, and cognition improved significantly in the group that received the magnifying aids [11]. This is why the goal of the current study was to find out whether additional RT in patients, who had first been administered their optimal visual aids and had used them, would have an additional positive effect on the outcome variables.

In the CG, there was a slight increase of median RS during placebo training $(8.7 \mathrm{wpm})$ and again during the later RT (by $10.6 \mathrm{wpm})$. However, the RS difference from t0 to $\mathrm{t} 2$ became statistically significant, which indicates additional effects of the RT. Even though solving crossword puzzles focuses on single words and does not require reading of continuous text (which was our reason to use this as placebo training), the activity necessary to solve this task may have prepared the way for improving RS.
Note that the training effect of RSVP on a screen transferred to reading printed text (page reading), the everyday reading task. The observation of a stable RS after the 6 weeks without training $(\mathrm{t} 3)$ shows that the training effect indeed generalized to an everyday reading situation.

Arditi (1999) [44] proposed "elicited" RSVP, where subjects can determine the duration of each word's presentation by themselves. In another study, improvement of RS by RSVP in normally sighted subjects was achieved by perceptual learning using a crowding [45] or a trigram letter recognition task [46].

A recent study applied two versions of training to the retinal periphery by RSVP with a trigram letter task (fixed vs. variable stimulus duration), and the effect of the training on RS was reported to be similar for both versions [47]. For patients with AMD, earlier studies using RSVP (without training) showed that RS was faster in RSVP compared with reading printed text $[27,37,38]$, or when the presentation rate varied with word length [28].

In our study, RSVP-RS was lower than RS of printed text at $t 1$ and $t 2$. The patients might have preferred reading comfort vs. RS during RSVP training, which can be especially important with the usually longer words in German. The advantage of printed text reading is the option to make regressive saccades in case of semantic uncertainty, and the preview benefit for guiding the next saccade.

A few studies applied RT in AMD; however, they were either limited by small sample sizes or by lacking control groups (for a review, see Pijnacker et al., 2011 [20]).

Chung (2011) [13] applied the interesting theoretical concept of perceptual learning with RSVP in a small sample of AMD patients for 6 weeks in weekly sessions of reading 300 sentences each and found an improvement of RS by $53 \%$.

Tarita-Nistor et al. (2014) [48] trained patients with central vision loss in a feasibility study at the acuity threshold - also a perceptual learning technique - which improved reading acuity and maximum RS.

The only RCT (using a repeated measurement crossover design) was performed by Seiple et al., (2011) [14], who compared 3 different training methods in AMD patients: (1) visual awareness and eccentric viewing, (2) oculomotor training, and (3) RSVP. They found an increase of RS only in the oculomotor training group. The lack of an effect of the RSVP training might be due to a different task: subjects had to report whether the read sentence made sense or not, which requires a cognitive judgment and may be less challenging. Another approach to improve reading by a customized reading rehabilitation program used stepwise progressive achievement of the goal included home training sessions [15], which showed improvement in reading performance and QoL in AMD patients. They improved every 2 weeks by approximately $15 \mathrm{wpm}$, which is a remarkably strong effect. It would 
have been interesting to see if the training effect remains stable after the end of the training period. A limitation of this training method might be the labor-intensive supervision through regular visits at the hospital.

Generally, the patients with low initial RS have more potential for improvement compared with those with a lesser degree of impairment. Besides a low RS at baseline, we did not find predictive factors that could identify patients who were likely to benefit from the training.

We also could not identify a mechanism that was responsible for the improvement of RS. It could be based on shortening of the fixation duration, as we observed in patients with Stargardt's disease performing RSVP training [12]. This hypothesis is supported by studies by Cheong et al. $(2007,2008)$ [49, 50], which showed significantly slower information transfer rates in AMD. Even though the reading time for a single word in the SLO did not change in our study, the fixation duration might have decreased in continuous text reading. It is also possible that the patients were motivated by the training to increase their daily reading time, which constituted additional unspecific RT.

Another approach to improve reading in AMD is eccentric viewing training (see review by Pijnacker et al., 2011) [20]. In the current study, this was not an issue, because all patients already used non-foveal fixation before the start of training.

\section{Eye movements and fixation}

Earlier studies have shown that fixation instability contributes to reading difficulties, which was shown in normal subjects [51] and in patients with AMD [52]; however, this was examined in patients with newly developed central scotoma or macular disease.

In the current study, the FSI was slightly subnormal and did not change between the measurements. This indicates that these patients were used to eccentric reading, which could be expected by their regular use of magnifying aids and the presence of an absolute central scotoma.

Therefore, fixation stability cannot be the main reason for the improvement of RS. This is an important finding, because we would have expected that the RSVP training could improve fixation stability. However, we have to consider that our patients started with nearly normal fixation stability, so that there was not much potential for improvement.

Interestingly, FSI correlated with RSVP-RS (at t1 and t2), which might be explained by the similar task-looking straight at the screen either for fixating the cross (FSI) or for single word reading during RSVP "without" reading eye movements. A more stable FSI could then be associated with a more stable fixation during the holding positions on the word during reading with RSVP.

\section{PRL location}

The location of the PRL (for fixation of a cross) did not change considerably between $\mathrm{t} 1$ and $\mathrm{t} 2$.

Most patients placed their PRL above the lesion. This is functionally more favorable than below regarding reading, because of a better orientation on the page.

There were only four outliers who changed their PRL by more than $6^{\circ}$. These patients might not have yet developed an established PRL for the cross.

It is known from several studies [53-56] that the use of a PRL depends on the task. For reading, a larger perceptual span is needed. One patient in the current study changed between paracentral fixation for the cross and eccentric fixation for words at $\mathrm{t} 1$ and $\mathrm{t} 2$. Central seeing islands in scotomas were described often in earlier studies, especially in dry AMD [41, 54, 57].

The idea behind RSVP is to prevent eye movements [26]. However, our patients made on average 5 fixations (median, IQR 4.25-6.25) per single word in the SLO. The high number of saccades while reading single words in the SLO indicates the diminished perceptual span in AMD patients, as was reported earlier [41, 42].

The improvement of RS during reading printed text cannot be explained by the eye movement variables during single word reading in the SLO, which did not change in a statistically significant way. This might be due to the fact that reading continuous text and single words are quite different tasks. In continuous text, reading the context triggers the next saccade to the next letter group or word. In this case, RS is mostly influenced by linguistic characteristics such as word length, word frequency, and familiarity with the topic. In addition, the patients' everyday reading situation is reading printed text with their magnifying aids. As this process is so different than reading single words, it is quite remarkable that the RSVP training transferred to printed text reading. We tentatively conclude that the fact alone that the patients had to read daily during the training enhanced other components of the reading process that do not depend strongly on eye movement parameters.

\section{Questionnaires about mood and QoL}

Severe impairment of emotional and cognitive well-being as indicated by MADRS and DEmTect questionnaires was an exclusion criterion for participation in this study. Consequently, the participating patients were only moderately or slightly impaired regarding emotional status and were in the normal range of cognitive status. Additionally, all patients had already received optimal visual aids and rehabilitation measures that had improved all these variables including QoL, which we showed in an earlier study [11]. Therefore, the potential for improvement was limited.

In our previous RCT pilot study [11], the waiting group received no intervention and deteriorated in emotional, 
cognitive, and social abilities, while the rehabilitation group showed the opposite effect.

\section{Emotional status (MADRS)}

The change of depression scores on the MADRS showed a significant difference between the groups with improvement in P-RTG, i.e., the RT could positively influence the emotional status. A mean difference of 4 points within several weeks is clinically meaningful. The combination of a clinical rating (MADRS) by a trained expert and self-rating by the patient is the gold standard for the evaluation of cognitive and emotional state, as this is more objective and recommended by clinical guidelines on depression [58]. The different development of the MADRS scores can be tentatively interpreted to indicate that the RT intervention might contribute to preventing secondary depression. The positive effect remained stable at $\mathrm{t} 3$. For future studies, a longer observation time would be desirable.

\section{QoL (IVI)}

Even though not much further improvement by the additional intervention of RT could be expected, it is worth mentioning that the complete IVI scores improved significantly in the TRTG from $\mathrm{t} 1$ to $\mathrm{t} 2$ and remained stable at $\mathrm{t} 3$.

Of the 20 questions regarding the functional score, only 2 were related to reading and in addition, these were too unspecific for our intervention. As our patients were already used to their appropriate visual aids, they already started with moderate reading performance. Therefore, the questions (no. 14 and no. 15) could not indicate a subjective improvement of reading by the RT: however, individual patient reports provided positive feedback about reading, such as "better," "more comfortable," and "is fun again."

The IVI emotional score, the questions which specifically refer to vision-related QoL, showed significant improvement in the $\mathrm{CG}$ from $\mathrm{t} 0$ to $\mathrm{t} 2$ and $\mathrm{t} 0 \mathrm{to} \mathrm{t} 3$, where the slight improvements during both training periods added to each other. However, no change occurred in the P-RTG, whose motivating "active" period possibly was only 6 weeks long. This should be compared to the CG with additional 6 weeks of placebo training that the patients enjoyed very much, which shows an unspecific effect. In contrast, pure waiting without intervention had a negative effect in our previous study [11].

In summary, we think that the complete IVI is more meaningful for the conditions of our study, which is confirmed by a recent study on the IVI questionnaire [59]. They reported that the summary IVI score is "likely adequate" for assessing outcomes of interventions, in contrast to subdividing the questionnaire into domains.

\section{Conclusions}

Original characteristics of our study are:

1) use of a control group,

2) training at home, which is especially valuable in AMD patients with limited mobility,

3) computer-based training, which allows independence and objective data mining. In addition, it saves costs by requiring a minimal number of visits to the clinic, and

4) a later follow-up examination after the end of training.

The study showed a significant improvement of RS by RSVP training for AMD patients, who have used optimal magnifying aids for a longer time and were therefore adapted to an established non-foveal PRL. The effect remained stable after the end of training, which indicates that the effect transferred to everyday life. A subgroup of 14 patients (38\%) clearly benefited in a clinically and practically meaningful way as they improved by $>10 \mathrm{wpm}$.

The significant difference in change of depression score between the groups hints at the possibility that RT could contribute to the prevention of secondary depression and mood impairment.

QoL improved during RT and remained stable.

Altogether, this study shows that it is beneficial for the patients to participate in RT after adaptation of visual aids, especially for those who start at a low RS.

Acknowledgments We thank Claudia Gehrlich, orthoptist, for her support in clinical examinations and data acquisition. We wish to thank our colleagues from private ophthalmology practices for the support in recruiting patients. We thank Manfred MacKeben, PhD, The Smith Kettlewell Eye Research Institute, San Francisco, USA, for helpful comments and editing support.

Funding This study was funded by the Baden-Wuerttemberg Department of Social and Labor Affairs (grant number Az 54-5431), the Intramural Research Programme Scholarship Medical Faculty University of Tübingen to KK (grant number IZKF 2014-2-05NR), the Charlotte and Tistou Kerstan Foundation, and the Lechler Foundation.

\section{Compliance with ethical standards}

Ethical approval All procedures performed in studies involving human participants were in accordance with the ethical standards of the institutional and/or national research committee and with the 1964 Helsinki declaration and its later amendments or comparable ethical standards.

The study was approved by the ethics committee of the University of Tübingen Medical Faculty (077/2012BO1). This article does not contain any studies with animals performed by any of the authors.

Informed consent Informed consent was obtained from all individual participants included in the study.

Conflict of interest The authors declare that they have no conflict of interest. 
Open Access This article is distributed under the terms of the Creative Commons Attribution 4.0 International License (http:// creativecommons.org/licenses/by/4.0/), which permits unrestricted use, distribution, and reproduction in any medium, provided you give appropriate credit to the original author(s) and the source, provide a link to the Creative Commons license, and indicate if changes were made.

\section{References}

1. Resnikoff S, Pascolini D, Etya'ale D et al (2004) Global data on visual impairment in the year 2002. Bull World Health Organ 82: 844-851

2. Casten RJ, Rovner BW, Tasman W (2004) Age-related macular degeneration and depression: a review of recent research. Curr Opin Ophthalmol 15:181-183

3. Rovner BW, Casten RJ (2002) Activity loss and depression in agerelated macular degeneration. Am J Geriatr Psychiatry 10:305-310

4. Brody BL, Gamst AC, Williams RA et al (2001) Depression, visual acuity, comorbidity, and disability associated with age-related macular degeneration. Ophthalmology 108:1893-1900 discussion 1900-1901

5. Horowitz A, Brennan M, Reinhardt JP, Macmillan T (2006) The impact of assistive device use on disability and depression among older adults with age-related vision impairments. J Gerontol B Psychol Sci Soc Sci 61:S274-S280

6. Rovner BW, Casten RJ, Tasman WS (2002) Effect of depression on vision function in age-related macular degeneration. Arch Ophthalmol 120:1041-1044

7. Hautzinger M (2015) Depressive Störungen. In: Maerker A (ed) Alterspsychotherapie und klinische Gerontopsychologie, 2nd edn. Springer, Berlin, pp 119-137

8. Grant P, Seiple W, Szlyk JP (2011) Effect of depression on actual and perceived effects of reading rehabilitation for people with central vision loss. J Rehabil Res Dev 48:1101-1108

9. Trauzettel-Klosinski S, Laubengeier C, Sadowski B, PietschBreitfeld B (2000) Reading ability with visual impairment: the significance of visual acuity and magnification requirement (article in German) [Lesefähigkeit bei Sehbehinderung: Die Bedeutung von Visus und Vergrößerungsbedarf]. Z Prakt Augenheilkd 21:529-533

10. Nguyen NX, Weismann M, Trauzettel-Klosinski S (2009) Improvement of reading speed after providing of low vision aids in patients with age-related macular degeneration. Acta Ophthalmol 87:849-853. https://doi.org/10.1111/j.1755-3768. 2008.01423.x

11. Mielke A, Wirkus K, Niebler R et al (2013) The influence of visual rehabilitation on secondary depressive disorders due to age-related macular degeneration. A randomized controlled pilot study (article in German)[Einfluss visueller Rehabilitation auf sekundäre depressive Störungen bei altersabhängiger Makuladegeneration]. Ophthalmologe 110:433-440. https://doi.org/10.1007/s00347012-2715-8

12. Nguyen NX, Stockum A, Hahn GA, Trauzettel-Klosinski S (2011) Training to improve reading speed in patients with juvenile macular dystrophy: a randomized study comparing two training methods. Acta Ophthalmol 89:e82-e88. https://doi.org/10.1111/j.1755-3768. 2010.02081.x

13. Chung STL (2011) Improving reading speed for people with central vision loss through perceptual learning. Invest Ophthalmol Vis Sci 52:1164-1170. https://doi.org/10.1167/iovs.10-6034

14. Seiple W, Grant P, Szlyk JP (2011) Reading rehabilitation of individuals with AMD: relative effectiveness of training approaches.
Invest Ophthalmol Vis Sci 52:2938-2944. https://doi.org/10.1167/ iovs.10-6137

15. Coco-Martín MB, Cuadrado-Asensio R, López-Miguel A et al (2013) Design and evaluation of a customized reading rehabilitation program for patients with age-related macular degeneration. Ophthalmology 120:151-159. https://doi.org/10.1016/j.ophtha. 2012.07.035

16. Kaltenegger K, Altpeter E, Cordey A et al (2016) Reading training in dry AMD improves reading ability and prevents secondary depression. Invest Ophthalmol Vis Sci 57:5168-5168

17. Trauzettel-Klosinski S, Kaltenegger K, Altpeter E et al (2016) Der Einfluss von Lesetraining auf die Lesefähigkeit, den emotionalen und kognitiven Status sowie die Lebensqualität bei Patienten mit altersabhängiger Makuladegeneration (AMD) - eine randomisierte und kontrollierte Studie - Abstract-Band DOG 2016. Ophthalmologe 113(Suppl 2):13-192. https://doi.org/10.1007/ s00347-016-0324-7

18. Hahn GA, Penka D, Gehrlich C et al (2006) New standardised texts for assessing reading performance in four European languages. $\mathrm{Br} \mathrm{J}$ Ophthalmol 90:480-484. https://doi.org/10.1136/bjo.2005.087379

19. Trauzettel-Klosinski S, Dietz K, IReST Study Group (2012) Standardized assessment of reading performance: the New International Reading Speed Texts IReST. Invest Ophthalmol Vis Sci 53:5452-5461. https://doi.org/10.1167/iovs.11-8284

20. Pijnacker J, Verstraten P, van Damme W et al (2011) Rehabilitation of reading in older individuals with macular degeneration: a review of effective training programs. Neuropsychol Dev Cogn B Aging Neuropsychol Cogn 18:708-732. https://doi.org/10.1080/ 13825585.2011.613451

21. Timberlake GT, Mainster MA, Peli E et al (1986) Reading with a macular scotoma. I. Retinal location of scotoma and fixation area. Invest Ophthalmol Vis Sci 27:1137-1147

22. Reinhard J, Messias A, Dietz K et al (2007) Quantifying fixation in patients with Stargardt disease. Vis Res 47:2076-2085. https://doi. org/10.1016/j.visres.2007.04.012

23. Steinman RM (1965) Effect of target size, luminance, and color on monocular fixation. J Opt Soc Am 55:1158-1164. https://doi.org/ 10.1364/JOSA.55.001158

24. Crossland MD, Rubin GS (2002) The use of an infrared eyetracker to measure fixation stability. Optom Vis Sci 79:735-739

25. Rohrschneider K (2004) Determination of the location of the fovea on the fundus. Invest Ophthalmol Vis Sci 45:3257-3258. https:// doi.org/10.1167/iovs.03-1157

26. Potter M (1984) Rapid serial visual presentation (RSVP): a method for studying language processing. In: Kieras D, Just M (eds) New methods in reading comprehension research. Erlbaum, Hillsdale, pp 91-118

27. Rubin GS, Turano K (1994) Low vision reading with sequential word presentation. Vis Res 34:1723-1733

28. Aquilante K, Yager D, Morris RA, Khmelnitsky F (2001) Lowvision patients with age-related maculopathy read RSVP faster when word duration varies according to word length. Optom Vis Sci 78:290-296

29. Montgomery SA, Asberg M (1979) A new depression scale designed to be sensitive to change. Br J Psychiatry J Ment Sci 134: 382-389

30. Craighead WE, Evans DD (1996) Factor analysis of the Montgomery-Asberg depression rating scale. Depression 4:3133. https://doi.org/10.1002/(SICI)1522-7162(1996)4:1<31::AIDDEPR3>3.0.CO;2-I

31. Galinowski A, Lehert P (1995) Structural validity of MADRS during antidepressant treatment. Int Clin Psychopharmacol 10:157-161

32. Kessler J, Calabrese P, Kalbe E, Berger F (2000) DemTect: a new screening method to support diagnosis of dementia. Psycho 26: 343-347 
33. Kalbe E, Kessler J, Calabrese P et al (2004) DemTect: a new, sensitive cognitive screening test to support the diagnosis of mild cognitive impairment and early dementia. Int J Geriatr Psychiatry 19: 136-143. https://doi.org/10.1002/gps.1042

34. Scheffels JF, Kräling H, Kalbe E, Kessler J (2018) Konversionen von kognitiven Screenings. Nervenarzt 89:1371-1377. https://doi. org/10.1007/s00115-018-0583-4

35. Nasreddine ZS, Phillips NA, Bédirian V et al (2005) The Montreal cognitive assessment, MoCA: a brief screening tool for mild cognitive impairment. J Am Geriatr Soc 53:695-699. https://doi.org/ 10.1111/j.1532-5415.2005.53221.x

36. Finger RP, Fenwick E, Marella M et al (2011) The impact of vision impairment on vision-specific quality of life in Germany. Invest Ophthalmol Vis Sci 52:3613-3619. https://doi.org/10.1167/iovs. 10-7127

37. Hassell JB, Weih LM, Keeffe JE (2000) A measure of handicap for low vision rehabilitation: the Impact of Vision Impairment profile. Clin Exp Ophthalmol 28:156-161

38. Lamoureux EL, Pallant JF, Pesudovs K et al (2006) The Impact of Vision Impairment questionnaire: an evaluation of its measurement properties using Rasch analysis. Invest Ophthalmol Vis Sci 47: 4732-4741. https://doi.org/10.1167/iovs.06-0220

39. Weih LM, Hassell JB, Keeffe J (2002) Assessment of the impact of vision impairment. Invest Ophthalmol Vis Sci 43:927-935

40. Hassell JB, Lamoureux EL, Keeffe JE (2006) Impact of age related macular degeneration on quality of life. Br J Ophthalmol 90:593596. https://doi.org/10.1136/bjo.2005.086595

41. Calabrèse A, Bernard J-B, Faure G et al (2014) Eye movements and reading speed in macular disease: the shrinking perceptual span hypothesis requires and is supported by a mediation analysis. Invest Ophthalmol Vis Sci 55:3638-3645. https://doi.org/10.1167/ iovs.13-13408

42. Crossland MD, Rubin GS (2006) Eye movements and reading in macular disease: further support for the shrinking perceptual span hypothesis. Vis Res 46:590-597. https://doi.org/10.1016/j.visres. 2005.05.015

43. Schotter ER, Angele B, Rayner K (2012) Parafoveal processing in reading. Atten Percept Psychophys 74:5-35. https://doi.org/10. 3758/s13414-011-0219-2

44. Arditi A (1999) Elicited sequential presentation for low vision reading. Vis Res 39:4412-4418

45. Treleaven AJ, Yu D (2018) Training peripheral vision to read: reducing crowding through an adaptive training method. Vis Res. https://doi.org/10.1016/j.visres.2018.05.009

46. Yu D, Cheung S-H, Legge GE, Chung STL (2010) Reading speed in the peripheral visual field of older adults: does it benefit from perceptual learning? Vis Res 50:860-869. https://doi.org/10.1016/j. visres.2010.02.006

47. Yu D, Legge GE, Wagoner G, Chung STL (2018) Training peripheral vision to read: boosting the speed of letter processing. Vis Res 152:51-60. https://doi.org/10.1016/j.visres.2017.06.005

48. Tarita-Nistor L, Brent MH, Steinbach MJ et al (2014) Reading training with threshold stimuli in people with central vision loss: a feasibility study. Optom Vis Sci 91:86-96. https://doi.org/10.1097/ OPX.0000000000000108

49. Cheong AMY, Legge GE, Lawrence MG et al (2007) Relationship between slow visual processing and reading speed in people with macular degeneration. Vis Res 47:2943-2955. https://doi.org/10. 1016/j.visres.2007.07.010

50. Cheong AMY, Legge GE, Lawrence MG et al (2008) Relationship between visual span and reading performance in age-related macular degeneration. Vis Res 48:577-588. https://doi.org/10.1016/j. visres.2007.11.022

51. Falkenberg HK, Rubin GS, Bex PJ (2007) Acuity, crowding, reading and fixation stability. Vis Res 47:126-135. https://doi.org/10. 1016/j.visres.2006.09.014

52. Crossland MD, Culham LE, Rubin GS (2004) Fixation stability and reading speed in patients with newly developed macular disease. Ophthalmic Physiol Opt 24:327-333. https://doi.org/10.1111/j. 1475-1313.2004.00213.x

53. Schuchard RA (2005) Preferred retinal loci and macular scotoma characteristics in patients with age-related macular degeneration. Can J Ophthalmol 40:303-312. https://doi.org/10.1016/S00084182(05)80073-0

54. Trauzettel-klosinski S, Tornow R-P (1996) Fixation behavior and reading ability in macular scotoma: assessed by Tuebingen manual perimetry and scanning laser ophthalmoscopy. Neuro-Ophthalmol 16:241-253. https://doi.org/10.3109/01658109609044632

55. Duret F, Issenhuth M, Safran AB (1999) Combined use of several preferred retinal loci in patients with macular disorders when reading single words. Vis Res 39:873-879

56. Safran AB, Duret F, Issenhuth M, Mermoud C (1999) Full text reading with a central scotoma: pseudo regressions and pseudo line losses. Br J Ophthalmol 83:1341-1347

57. Sunness JS, Rubin GS, Zuckerbrod A, Applegate CA (2008) Foveal-sparing scotomas in advanced dry age-related macular degeneration. J Vis Impair Blind 102:600-610

58. DGPPN, BÄK, KBV, AWMF (Hrsg) (2015) für die Leitliniengruppe Unipolare Depression*. S3-Leitlinie/Nationale VersorgungsLeitlinie Unipolare Depression - Langfassung, 2. Auflage. Version 5. https://doi.org/10.6101/AZQ/000364. www. depression.versorgungsleitlinien.de. Accessed 09 April 2019. (*Organisationen, die in der Leitliniengruppe kooperierten: DGPPN, BÄK, KBV, AWMF, ACKPA, AkdÄ, BPtK, BApK, DAGSHG, DEGAM, DGPM, DGPs, DGRW, BDK, BDP, BPM, BVDN, BVDP, BVVP, CPKA, DÄVT, DFT, DGGPP, DGPT, DGVT, DPG, DPV, DPtV, DVT, GwG, Stiftung Deutsche Depressionshilfe)

59. Goldstein JE, Fenwick E, Finger RP et al (2018) Calibrating the Impact of Vision Impairment (IVI): creation of a sample-independent visual function measure for patient-centered outcomes research. Transl Vis Sci Technol 7:38. https://doi.org/10.1167/tvst.7.6.38

Publisher's note Springer Nature remains neutral with regard to jurisdictional claims in published maps and institutional affiliations. 